ESJ Humanities

\title{
Potentiels agronomiques des Niayes de Mboro (littoral Nord du Sénégal) et risques de dégradation mécanique par ensablement : approche par analyse géomorphologique des enjeux socio-économiques
}

\author{
Dr. Mouhamadou Bassirou Seck \\ Pr. Boubou Aldiouma Sy \\ Laboratoire Leïdi «Dynamiques des Territoires et Développement » (DTD), \\ Université Gaston Berger, Sénégal
}

Doi:10.19044/esj.2021.v17n20p140

Submitted: 19 April 2021

Accepted: 18 June 2021

Published: 30 June 2021
Copyright 2021 Author(s)

Under Creative Commons BY-NC-ND

4.0 OPEN ACCESS

Cite As:

Bassirou Seck M. \& Aldiouma Sy B. (2021). Potentiels agronomiques des Niayes de Mboro (littoral Nord du Sénégal) et risques de dégradation mécanique par ensablement : approche par analyse géomorphologique des enjeux socio-économiques. European Scientific Journal, ESJ, 17(20), 140. https://doi.org/10.19044/esj.2021.v17n20p140

\section{Résumé}

La côte de Mboro, partie intégrante de la Grande Côte du Sénégal, est un creuset de territoires porteurs d'enjeux socio-économiques. Ces territoires sont formés d'écosystèmes supports de l'économie agricole maraîchère en sus des activités inhérentes à la littoralisation de la vie. C'est un espace qui est soumis aux facteurs de la morphodynamique du système de dunes littorales. Ce sont des facteurs agents du risque vecteurs de la dynamique d'ensablement en cours. L'objectif de cet article est de déterminer la granulométrie du substrat dunaire afin d'apprécier sa sensibilité au vent, d'une part, et de mesurer les enjeux socio-économiques du potentiel agronomique maraîcher à Mboro, d'autre part. Dans ce cadre, la méthodologie est basée sur un échantillonnage de sol, l'analyse granulométrique par tamisage et des enquêtes socio-économiques in situ. Compte tenu de la sensibilité granulaire des sédiments à la déflation face au potentiel agronomique des Niayes ${ }^{1}$ à Mboro, une recherche axée sur l'intensité du risque de dégradation mécanique des profils pédologiques est justifiée dans cet environnement aérologique favorable à la déflation éolienne et sur l'activité économique autour des

\footnotetext{
${ }^{1}$ Bas-fonds entre le système de dunes littorales et ogoliennes (cuvettes maraichères).
} 
Niayes. Les résultats ont montré une granulométrie globalement fine, soit plus $90 \%$ du poids des échantillons traités susceptibles au mode de transport par saltation et par suspension éolienne, et un potentiel cultural de quelque 14 variétés. Ce qui témoigne des enjeux de la recherche géomorphologique pour les actions d'aménagement du territoire.

Mots-clés: Ensablement, Mboro, Niayes, granulométrie, risque morphogénique, enjeux socio-économiques

\title{
Agronomic Potential of Niayes's Mboro (Northern Coast of Senegal) and Risks of Mechanical Degradation by Silting Up: Approach by Geomorphological Analysis of Socio-economic Issues
}

\section{Dr. Mouhamadou Bassirou Seck \\ Pr. Boubou Aldiouma Sy}

Laboratoire Leïdi « Dynamiques des Territoires et Développement » (DTD), Université Gaston Berger, Sénégal

\begin{abstract}
The coast of Mboro, Great Coast of Senegal, is a melting pot of territories with socio-economic challenges. These territories are made up of ecosystems that support the agricultural market gardening economy in addition to the activities inherent in the coastalization of life. It is a space which is subject to factors of the morphodynamics of the coastal dune system. These are risk factors vectors of the current sand-silting dynamic. This paper focuses on sampling and determining the granulometry of the dune substrate in order to assess its sensitivity to the wind, on the one hand, and to measure the socio-economic issues of the agronomic market gardening potential in Mboro, on the other hand. In this context, the methodology targets soil sampling, particle size analysis by sieving, and socio-economic surveys in situ. Given the granular sensitivity of the sediments to deflation in the face of the agronomic potential of Niayes at Mboro, research focused on the intensity of the risk of mechanical degradation of soil profiles is justified in the aerological environment favorable to wind deflation and on the economic activity around the Niayes. The results showed a medium to fine grain size, i.e., over $90 \%$ of the weight of treated samples susceptible to the saltation and wind suspension mode of transport, and a crop potential of some 14 varieties. This testifies to the challenges of geomorphological research for land use planning actions.
\end{abstract}


Keywords: Silting, Mboro, Niayes, granulometry, morphogenic risk, socioeconomic issues

\section{Introduction}

La côte est un écosystème très dynamique du fait de la composition du système en place. La résultante de cette dynamique fournit un écosystème qui fonctionne avec des emboitements, des répercussions et des résonnances assez significatives entre les différents éléments constitutifs de la côte. Les cuvettes maraîchères des Niayes subissent un ensablement hérité du matériau dunaire très mobile sous l'action éolienne. Au-delà de l'aspect mécanique, la déflation éolienne provoque un phénomène morphogénique par l'accrétion du matériau dunaire sur les sols hydromorphes organiques des Niayes (Niang, 2017). C'est un risque qui compromet le potentiel agronomique des sols des Niayes car les particules de sable d'apport dunaire sont issues du cordon blanc adjacent à la Haute plage. L'altération de sols organiques des Niayes (Sy, 2013), unité morphopédologique représentant une dépression entre les dunes jaunes et les dunes ogoliennes et marquée par une forte hydromorphie, devient une réalité. Sur le plan morpho-dynamique, le secteur de Mboro est une frange côtière du littoral nord sénégalais appelé Grande Côte marquée par une succession de générations de dunes littorales sensibles au transport éolien. Les dunes blanches très envahissantes supportent des sols minéraux bruts non climatiques très peu évolués donc avec de faibles teneurs en matières organiques et colonisés par des espèces steppiques, les espèces Halospammophytes (Sy, 2009). Les dunes jaunes couvertes de buissons, souvent ravivées et dont la largeur peut atteindre $4500 \mathrm{~m}$, se rétrécissent considérablement à l'aval de Mboro (Fall, 1986). Ces unités dunaires jaunes et blanches sont en effet, pourvoyeurs de quantités considérables de sédiments dont le bilan massique extrapolé en gr./ha/mois sur un dispositif de trappe à sable est estimé à plus de 730,37 tonnes sur Mboro en saison sèche chaude (Seck, 2019). C'est un potentiel érosif menaçant en direction des Niayes de Mboro.

La problématique réside dans les enjeux économiques des Niayes du Sénégal, en termes d'aptitudes agricoles, qui fournissent plus de $60 \%$ de la production maraîchère nationale (Ndao, 2012). Cependant, le phénomène d'ensablement reste une contrainte majeure à cette production. Cet état de fait met au centre des préoccupations les enjeux de la recherche en géomorphologie. Le postulat est que la sensibilité des grains à la déflation est un risque systémique face aux enjeux socio-économiques des territoires de la côte de Mboro.

Mboro est une commune localisée administrativement dans le département de Tivaouane de la région de Thiès. 




Figure 1. Localisation du secteur côtier de Mboro, littoral Nord du Sénégal

Mboro bénéficie d'une frange côtière qui lui permet de superposer plusieurs paysages notamment agricoles. Une palette importante de cet écosystème est constituée, en effet, d'espaces maraîchers et de territoires dont les activités sont fortement liées à la côte. Des terroirs villageois s'organisent autour de localités qui ont subies les différents travaux de terrain. Il s'agit des localités de Mboro-sur-mer, de Golgaïndé, de Bono, de Khondio. 




Figure 2. Les sites et paysages agricoles

Cette contribution cible la sensibilité granulométrique du substrat de la côte de Mboro et l'appréciation des enjeux socio-économiques autour des Niayes de Mboro, ce qui justifie la recherche empirique pour atteindre ces objectifs.

\section{Méthodologie}

La méthodologie tourne autour de deux grands axes. Il s'agit de l'échantillonnage, de l'analyse granulométrique et des enquêtes socioéconomiques.

\section{1. Échantillonnage et analyse granulométrique}

L'analyse granulométrique d'échantillons pris dans le secteur de Mboro détermine le calibrage des sédiments prélevés dans les différentes séquences. Elle s'est déroulée en laboratoire par méthode de tamisage sur la Basse Plage, la Haute Plage et le Cordon vif respectivement T1P1N1, T1P2N1 et T1P3N1. La campagne de prélèvement a été conduite à Mboro-sur-mer. Le prélèvement a été fait en surface, soit un seul niveau $N 1$, un seul transect a été réalisé et codé $T 1$, trois puits réalisés $P 1, P 2, P 3$. La base de correspondance 
entre les textures et les mailles des tamis (Sy, 2009) est la suivante : les argiles (diamètre > 0,02 mm), le Limon Fin $(0,02$ à $0,05 \mathrm{~mm})$, le Limon Grossier $(0,05$ à $0.06 \mathrm{~mm})$, le Sable Très Fin $(0,06$ à $0,12 \mathrm{~mm})$, le Sable Fin $(0,12$ à $0,20 \mathrm{~mm})$, le Sable Moyen $(0,20$ à $0,71 \mathrm{~mm})$, le Sable Grossier $(0,71$ à $1 \mathrm{~mm})$ et Sable Très Grossier ( 1 à $2 \mathrm{~mm}$ ).

Le protocole de laboratoire se base sur une analyse granulométrique par tamisage aux normes de référence NFP94-056 (tamisage à sec après lavage) pour déterminer le calibre des populations de grains et procède par :chasser les intrus consiste à séparer les sols des débris de végétaux en particulier la litière très abondante à Mboro, à l'aide d'un tamis ; - répartition des échantillons en deux bols pour chacun des trois échantillons qui sont à ré-échantillonner pour donner 6 bols dont 3 pour l'étude de la teneur en eau et 3 autres pour l'analyse granulométrique ; - l'analyse proprement dite de notre échantillon de sol qui, étant humide, nécessite un pesage initial pour avoir le poids net des sédiments à sec. Pour le Bol 1, réservé à l'analyse granulométrique, il passe par diverses opérations : - pesage initial à l'aide de la balance électrique de précision (Photo 1 ) afin de déterminer le poids initial ; - lavage avec tamis $\mathbf{0 , 8 0} \mathbf{~ m m}$; - séchage à l'étuve (Photo 2) pendant 24 heures ; - tamisage après 15 minutes d'attente ; - détermination de la granulométrie. Le Bol 2 est réservé à l'analyse de la teneur en eau et il passe également par divers traitements : - pesage initial ; - passage à l'étuve pendant 24 heures ; - pesage définitif.

Calcul de la masse sèche $: \mathbf{M s}=\mathbf{M} /(\mathbf{1}+\mathbf{w})$ avec $\mathbf{w}$ (teneur en eau) et $\mathbf{M}$ (masse de l'échantillon humide), d'après Myriam Olivier et Ali Mesbah (2016). La balance électrique de précision effectue les pesages nécessaires (Photo1). Les sédiments sont nettoyés, avec l'étuve de séchage, de toute teneur en eau susceptible de fausser le pesage du matériau sec. Une colonne de 15 tamis a été utilisée (Photo 2). 




Photo 1 et 2. Balance électrique de précision et Étuve de séchage (Seck, 2019)

Les résultats sont notés sur une feuille d'assignation où sont présentés les paramètres statistiques recommandés par Fournier et al. (2012). Il s'agit de :

- Refus en \% et refus cumulé ce sont les masses de granulat retenues sur chaque module après vibration (en \% et cumulées) ;

- Passant en \% désigne les masses de granulat qui passent entre les mailles des différents modules. Ces informations sont aussi analysées par des courbes granulométriques. Il s'agit des courbes cumulatives où les fréquences cumulées sont utilisées pour former des indicateurs graphiques et traduire les informations sédimentologiques. Pour les courbes granulométriques sous Excel, les pourcentages des tamisats cumulés sont portés sur une échelle semi-logarithmique. Sur l'axe des abscisses, sont portées les dimensions des mailles, à l'échelle logarithmique et sur l'axe des ordonnées à l'échelle arithmétique sont portés les pourcentages. Outre l'analyse granulométrique, la méthodologie a recours à une enquête socio-économique sur les enjeux de l'économie agricole des Niayes de Mboro. 


\subsection{Enquêtes socio-économiques dans les Niayes de Mboro}

Il convient d'interroger des personnes ressources sur la base d'un questionnaire. La Population cible (N) est l'ensemble de tous les maraîchers des Niayes des villages de Bono et de Golgaïndé en activité pendant la période de l'enquête (16 Avril 2019). Elle intègre la population active du département de Tivaouane estimée à $24,2 \%$ de la population totale de la commune de Mboro de 28888 habitants (ANSD, 2015). Etant donné que la majorité de la population de Mboro est composée d'agriculteurs notamment maraîchers, à titre indicatif, ces statistiques donnent une idée de la population ciblée par l'enquête. L'Échantillon (n) au jugé a ciblé quelque 30 maraîchers interrogés sur l'ensemble de tous les maraîchers des Niayes des villages de Bono et de Golgaïndé, qui sont en activité pendant le temps de l'enquête (16 avril 2019). C'est un échantillonnage au jugé qui, hypothétiquement et au regard des statistiques (plus de la majorité de la population active étant composée de maraichers), peut être estimé à $1 / 10^{\mathrm{e}}$ de la population agricole maraîchère. Il offre une base d'étude cohérente des questions liées aux impacts socioéconomiques des stratégies de protection même si théoriquement, c'est un échantillon difficile à chiffrer suivant les paramètres mis en exergue : maraîchers des villages de Bono et de Golgaïndé en activité pendant l'enquête. La Séquence temporelle ou périodicité : l'étude porte sur une analyse comparative des facteurs et des conséquences socio-économiques des aménagements afin d'apprécier les véritables enjeux de la protection des Niayes. Pour ce faire, les questions ont porté sur les indicateurs socioéconomiques dans la séquence 2005-2019, qui correspond au deuxième aménagement de la bande de filao à Mboro survenu en 2005. La Localisation désigne les sites retenus qui sont les villages de Golgaïndé et de Bono très caractéristiques des conditions physiques de la côte : présence de Niayes, de dunes, du maraîchage, de la bande de filao, de la végétation naturelle, etc. Ces paramètres permettent de justifier le choix porté sur ces villages susceptibles d'abriter une telle étude avec des personnes ressources disponibles. Le questionnaire est confectionné sous Le Sphinx Plus ${ }^{2}$ et les résultats qui y sont traités sont des données statistiques importantes.

\section{Analyse des résultats}

Les résultats obtenus fournissent des données sur le calibre des particules de sable analysées et des indicateurs socio-économiques sur les activités maraîchères. La sensibilité des sédiments de la côte de Mboro se traduit en risque face aux territoires porteurs d'enjeux socio-économiques.

\subsection{Risque érosif lié à la sensibilité granulaire des sédiments}

L'analyse granulométrique établit une trame sédimentaire de différents calibres. Il est à rappeler que les sédiments moyens, fins et très fins 
sont susceptibles au transport éolien par suspension et par saltation (Thiaw, 2018). En outre, Tangara (2010) estime que la totalité des vents sont compétents dans les actions de sapement, de charriage, de transport et de dépôt des particules de sable de la côte Nord du Sénégal. Les résultats bruts sont des refus et des tamisats exprimés en fréquences.

Structure granulométrique de la Basse Plage. Les statistiques permettent d'extraire les informations pertinentes pour l'échantillon de l'infratidale. Graphiquement, la distribution des textures du sol de l'estran peut être décrite par la Figure 3.

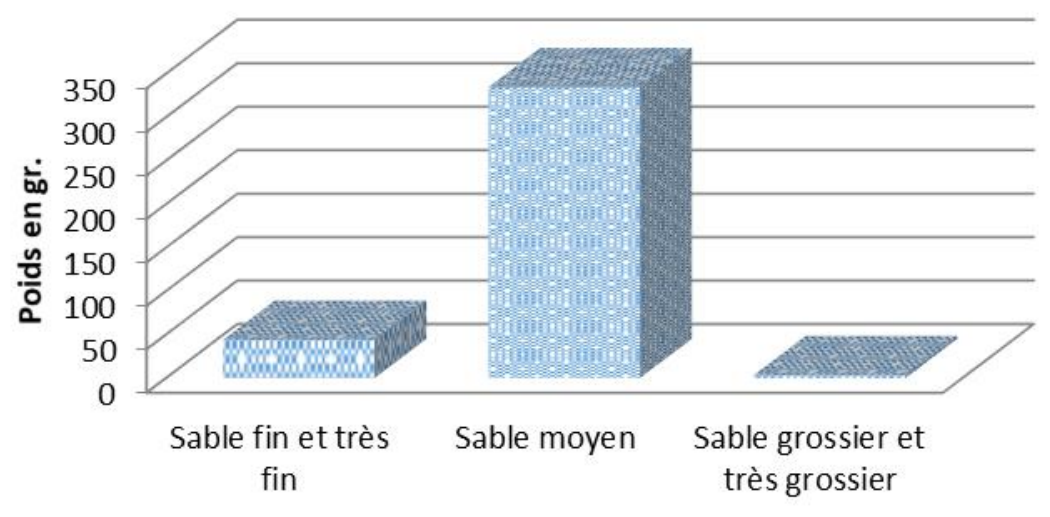

Textures de la Basse Plage

Figure 3. Structure granulométrique des textures de la Basse Plage

La structure granulaire de la Basse plage permet de distinguer une prédominance très nette de la classe des grains de calibre moyen sur l'étendue des observations. Les grains compris entre $0,20 \mathrm{~mm}$ et $0,71 \mathrm{~mm}$ occupent une part prépondérante avec $87,6 \%$ des observations pour 334,5 g. Les caractéristiques dimensionnelles de ces sédiments présagent une compétence des vents supérieurs à $5,63 \mathrm{~m} / \mathrm{s}$ (Niang, 2017). S'ensuivent les grains de diamètre fin et très fin. Très susceptibles au transport éolien, ils concentrent $11,5 \%$ des granulats analysés, soit 43,9 g. Leur existence permet d'apprécier la force érosive à mobiliser et à transporter les particules de sable. Enfin, les sables grossiers et très grossiers ne représentent que $0,9 \%$. La courbe cumulative illustre cette situation (Figure 4). 




Figure 4. Courbe cumulative des fréquences granulométriques de la Basse Plage

La courbe des fréquences cumulées des diamètres des sédiments révèlent deux tendances globales sur la Basse Plage. Les sédiments inférieurs à $0,71 \mathrm{~mm}$ de diamètre dégagent une situation de cumul croissante. Ce sont les sédiments de calibre très fin, fin et moyen qui prédominent dans l'échantillon. La seconde situation est une tendance stable qui se manifeste à partir de 0,71 mm de diamètre. Minoritaires dans l'échantillon de la Basse Plage, les sables grossiers sont pour autant résistants face à la dynamique érosive. Mieux, la distribution granulométrique des sédiments de l'échantillon de la Basse Plage permet d'apprécier la répartition des granulats selon le calibre (Figure 5).



Figure 5. Courbe granulométrique de l'échantillon de la Basse plage

Le profil de la Basse Plage est essentiellement centré entre $0,1 \mathrm{~mm}$ et $0,63 \mathrm{~mm}$, révélant la prédominance des particules de sable de diamètre moyen, fin et très fin ; la fraction grossière étant minimale. Les grains de taille 
inférieure à $0,20 \mathrm{~mm}$ sont très sensibles à la déflation éolienne. Une situation quasi similaire s'observe avec les autres séquences de la plage de Mboro.

Structure granulométrique de la Haute Plage. Les statistiques de la Haute Plage sont traitées et représentées à travers la Figure 6.

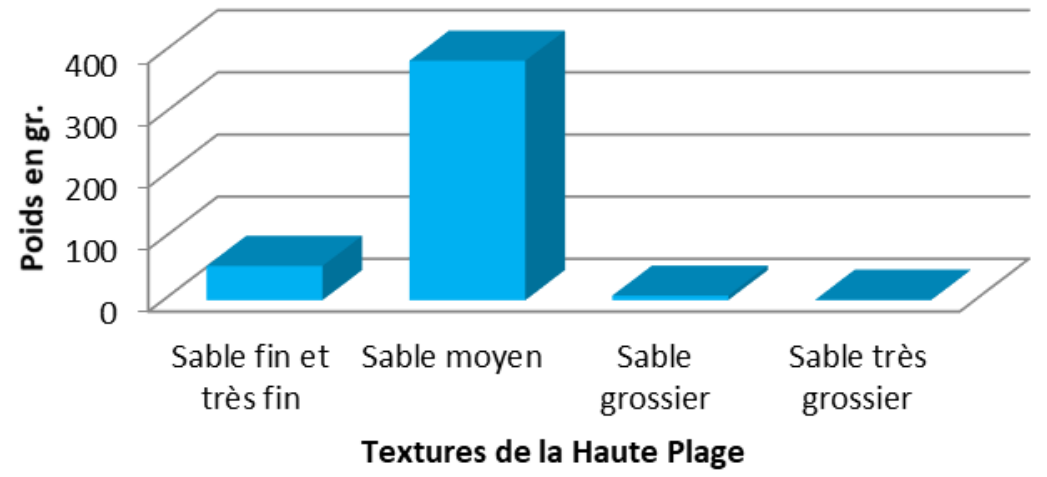

Figure 6. Structure granulométrique des textures de la Haute Plage

La Figure 6 montre la distribution dimensionnelle des granulats de la Haute Plage. Les sables de diamètre moyen couvrent une fréquence considérable, soit 85,82\% (un poids de $385.5 \mathrm{~g}$ ) sur la totalité des observations. La prédominance des sables moyens est significative. Ils sont suivis de loin par les sables fins et très fins avec un cumul des fréquences de $12,4 \%$, soit $55,2 \mathrm{~g}$ du poids. Ce sont des calibres très sensibles à la déflation. La courbe cumulative (Figure 7) caractérise cette domination des particules de diamètre fin à moyen.



Figure 7. Courbe cumulative des fréquences granulométriques de la Haute Plage

Le troisième groupe de sédiments s'insère dans la deuxième tendance de la courbe et comprend les sables grossiers, soit de $0,71 \mathrm{~mm}$ à $1 \mathrm{~mm}$. Ils 
représentent $1,63 \%$ des observations. Les sédiments très grossiers sont résiduels avec une représentativité de $0,15 \%(0,65 \mathrm{~g})$. La courbe granulométrique de la Haute Plage montre la distribution autour des sédiments fins et moyens (Figure 8).

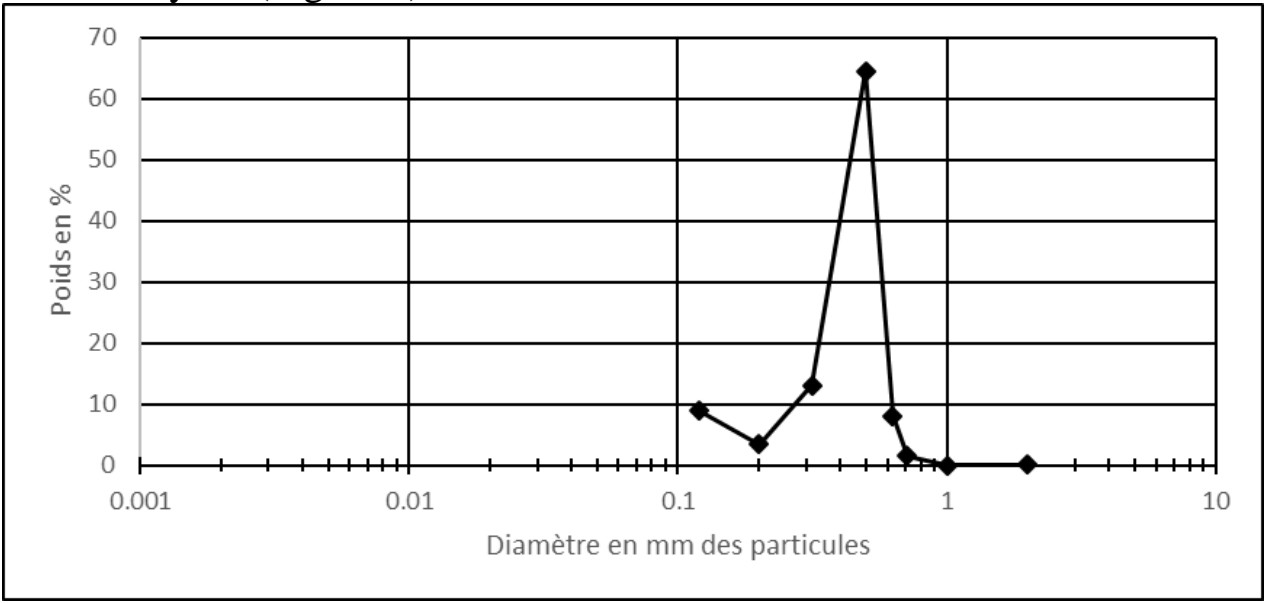

Figure 8. Courbe granulométrique de l'échantillon de la Haute Plage

La distribution granulométrique de l'échantillon de la Haute Plage montre une structure non étalée témoin de l'origine identique des particules de sable. C'est une structure centrée sur la trame sédimentaire comprise entre 0,1 $\mathrm{mm}$ et $1 \mathrm{~mm}$ de diamètre, ce qui traduit la dominance des sables de taille moyenne et fine susceptibles à la mobilisation éolienne. La fraction grossière est toujours minimale.

Structure granulométrique du Cordon vif. Le cordon vif littoral ou Supratidale est la séquence supérieure de la plage atteinte exceptionnellement par les hautes marées. C'est la partie qui représente le front aménagé de la bande de filao. Les granulats prélevés sur cette portion de la plage sont analysés et représentés. La représentativité des populations granulométriques peut être ressortie sur un diagramme (Figure 9). 


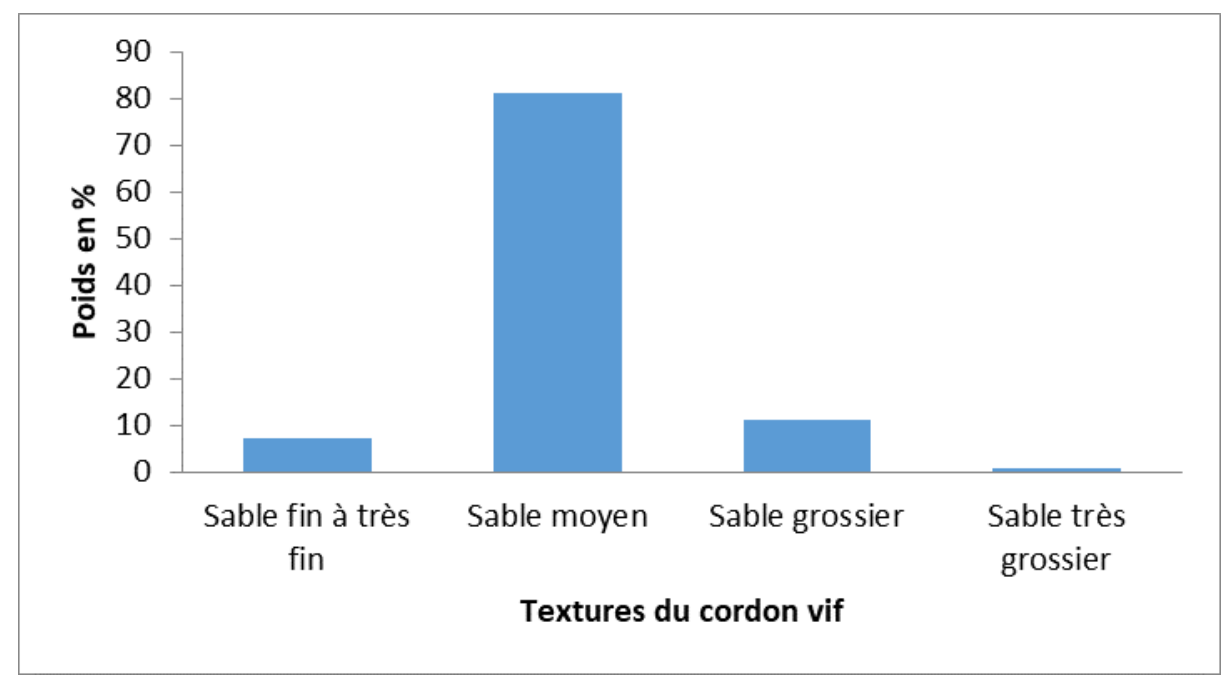

Figure 9. Structure granulométrique des textures du cordon littoral

La distribution est assez inégale des textures de sable partant des grains de $0,12 \mathrm{~mm}$ de diamètre (STF) à $2 \mathrm{~mm}$ de diamètre (STG). Sont représentées les textures suivantes par ordre d'importance : les sables moyens avec 81,2\% des observations, les sables grossiers avec $11 \%$ des observations. Leur représentativité est caractéristique de la granulométrie du cordon vif. C'est la différence notable avec la texture générale de l'estran. Les sables fins et très fins font $7 \%$ des observations. Ce qui laisse entrevoir une forte sensibilité à la mobilisation et au transport des sédiments par le fluide éolien. Les sables très grossiers sont résiduels $(0,8 \%)$. La courbe cumulative décrit quasiment la même situation (Figure 10).



Figure 10. Courbe cumulative des fréquences granulométriques du Cordon Vif 
La courbe décrit une situation plutôt similaire observée sur la Basse et la Haute plage, soulignant la parenté génétique des sédiments. En effet, une première tendance progressive des cumuls montre une dominance des sables de diamètre inférieur à $0,71 \mathrm{~mm}$, donc moyens, fins et très fins. La deuxième tendance part des sédiments de calibre supérieur à $0,71 \mathrm{~mm}$ jusqu'au millimètre et même entre 1 et $2 \mathrm{~mm}$. D'où la spécificité de cet échantillon du cordon vif avec une présence de sable très grossier plus résistant alors à la force éolienne. Cette situation est appréciable sur la courbe de distribution granulométrique (Figure 11).



Figure 11. Courbe granulométrique de l'échantillon du Cordon blanc vif

La structure de la courbe (Figure 11) révèle une meilleure stabilité : elle est bimodale centrée sur les sables de calibre moyen et grossier. La dominance de la fraction moyenne est évidente. Cependant, les sables grossiers représentent $11 \%$ de l'échantillon, quantité non négligeable. Il existe aussi une fraction très grossière dont le diamètre est supérieur à $1 \mathrm{~mm}$. Ces graphiques traduisent une comparaison interne des différentes textures des séquences de la plage : Basse, Haute et Cordon vif, ce qui justifie l'appréciation de la structure générale des séquences (Figure 12). 




Figure 12. Histogramme des fréquences granulométriques de la plage de Mboro

A la lumière de ces données lithologiques et sur la base que les sédiments de calibre moyen et fin sont très sensibles à la déflation éolienne (Seck, Sy \& Sy, 2020), il est admis un risque érosif important sur les espaces de culture. Dans ce cadre, le risque morphogénique est une prédisposition d'un milieu géographique à être altéré par le système morphogénique (altération mécanique du vent sur le substrat dunaire) (Seck, 2019). Les débits solides potentiellement mobilisables sont des données objectives caractéristiques d'un risque érosif. Les particules de sable érodées par le fluide éolien à la surface des dunes (revers du cordon blanc) sont mobilisées et transportées vers les dépressions. Les Niayes piègent ainsi les débits solides éoliens, fonctionnant comme des environnements sédimentaires : ensablement des cuvettes maraîchères. Les dépôts de sable des dunes sur ces espaces agricoles altèrent la composition physique des sols, ce qui dégrade progressivement le potentiel agronomique des cuvettes maraichères (Niayes). Le matériau d'un sol minéral brut non climatique ensevelit les sols hydromorphes lourds (forte teneur en argile et limon) et en matières organiques des Niayes. Les Niayes structurent ainsi des territoires porteurs de l'économie agricole à enjeux multiples.

\subsection{Les indicateurs socio-économiques: des territoires porteurs d'enjeux}

Les données quantitatives recueillies lors de l'enquête sont des variables mesurables. Le dépouillement permet d'apprécier ces enjeux. La variété et l'évolution de la qualité des cultures sont ainsi prises en compte par le questionnaire. 
«Quels sont les types de culture pratiqués? » est la question 17/23 soumise à la population maraîchère. Les trente (30) réponses recueillies ont permis de dresser une liste qui contient les variétés agronomiques généralement pratiquées dans les Niayes de Mboro. Chaque interlocuteur a dressé son répertoire et le dépouillement met en exergue 14 variétés culturales : pomme de terre, aubergine (douce et amère), piment, chou, patate, oignon, salade, concombre, navet, tomate, arachide, carotte, poivre et haricot. Les réponses peuvent faire l'objet de classification pour trouver la fréquence des différentes cultures. La culture de pomme de terre prédomine, soit 12,8\% de l'effectif total des cultures. Le piment (ordinaire, « piment salade »), le navet, le chou, l'oignon et la carotte sont également des cultures très représentatives. S'ensuivent les cultures de l'aubergine (douce et amère), de la patate et du concombre qui représentent respectivement $9 \%, 6 \%$ et 5,4\% des cultures. $\mathrm{La}$ salade, la tomate, l'arachide, le poivron et le haricot suivent.

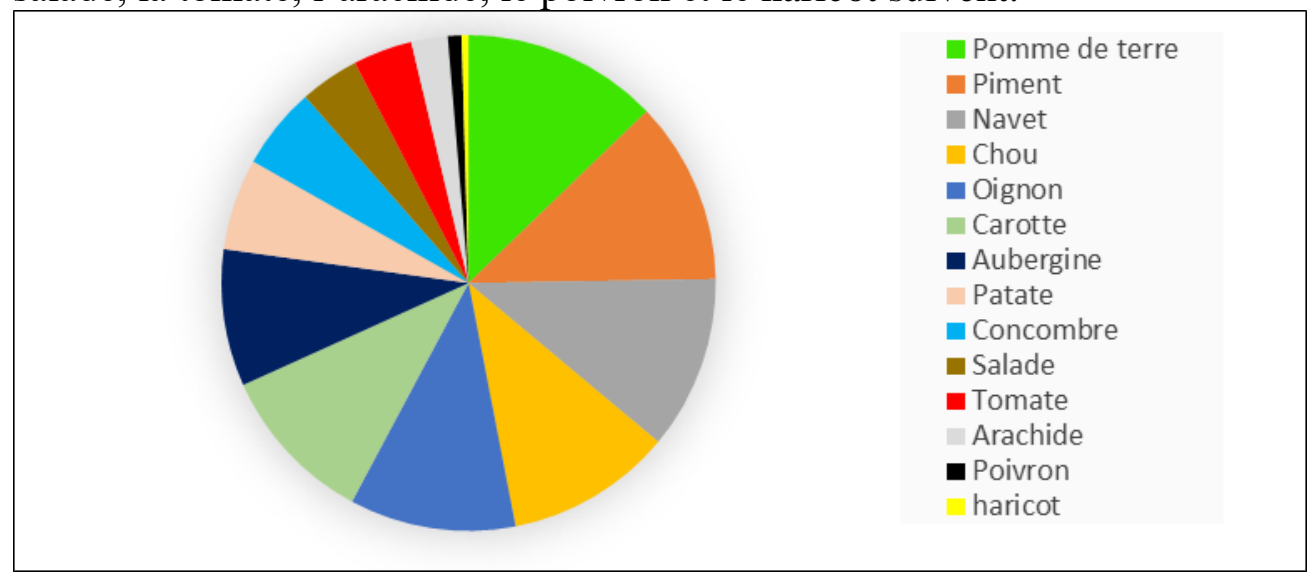

Figure 13. Fréquence des cultures en rapport aux réponses fournies

Dans quasiment tous les champs visités pendant les activités de terrain, il existe des plans et des tas de récolte de pomme de terre. Aussi, le navet, le chou, le piment, l'oignon, la carotte, l'aubergine, la patate et le concombre sont assez bien illustrés dans les champs visités. Ces cultures sont assez prisées dans le marché local et régional (Thiès, Tivaouane, ...) et surtout dans la capitale Dakar. Elles sont pratiquées par plus de $35 \%$ des maraîchers interrogés. Les plantations sont caractéristiques d'un regain d'activité agricole. 


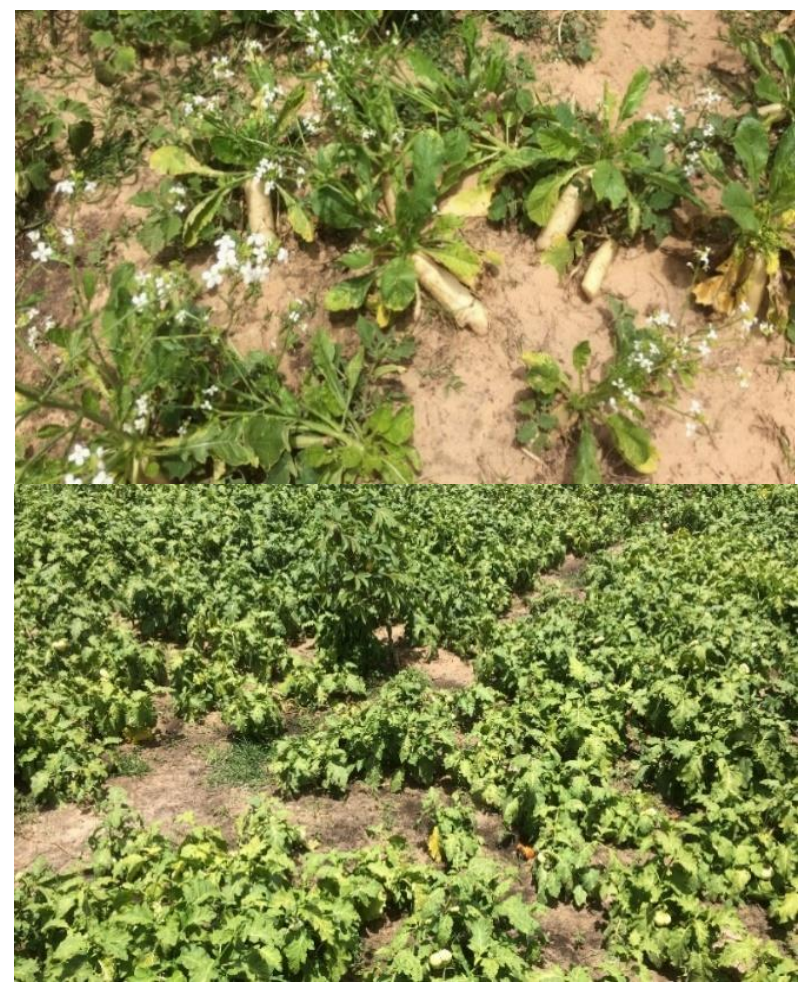

Photo 3 et 4. La culture du navet et de l'aubergine (Seck, 2019)

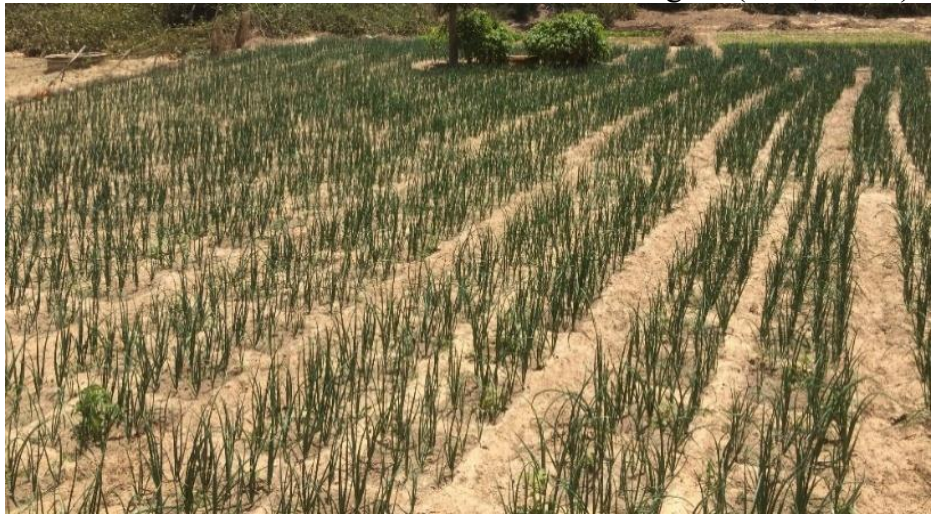

Photo 5. Plan d'oignon (Seck, 2019)

Plus de $76 \%$ des personnes interrogées pratiquent cette culture. Le navet (Brassica rapa) est une plante herbacée de la famille des Brassicacées. C'est une plante potagère consommée comme légume. Le marché sénégalais en est inondé et les maraîchers s'adonnent à cette culture au regard de sa rentabilité. La Photo 4 illustre un plan de la culture de l'aubergine où deux types sont exploités dans les champs visités : l'aubergine amère ou aubergine africaine et l'aubergine douce. La première est ainsi nommée à cause du goût amer. Les pratiques culinaires sénégalaises s'accommodent aussi bien de l'une 
que de l'autre. Les menus sénégalais sont très souvent ornés de ces deux types d'aubergine et en même temps.

Les plans d'oignon sont disposés en sillons (Photo 5) ; l'oignon est une culture très bien représentée dans les champs de maraichage de Golgaïndé, de Mboro-sur-mer, de Bono. La protection des espaces de culture est d'un enjeu socio-économique important : protéger le patrimoine cultural des Niayes de Mboro. La qualité des cultures ici exposée est une conséquence des aménagements de protection. La population explique cette situation par deux ensembles de réponses : la réponse "sol régénéré et cultures protégées de l'ensablement » devient plus explicite avec la réponse "sol régénéré et cultures protégées contre le vent, la chaleur et le sable dunaire » (Photos 6 et 7).

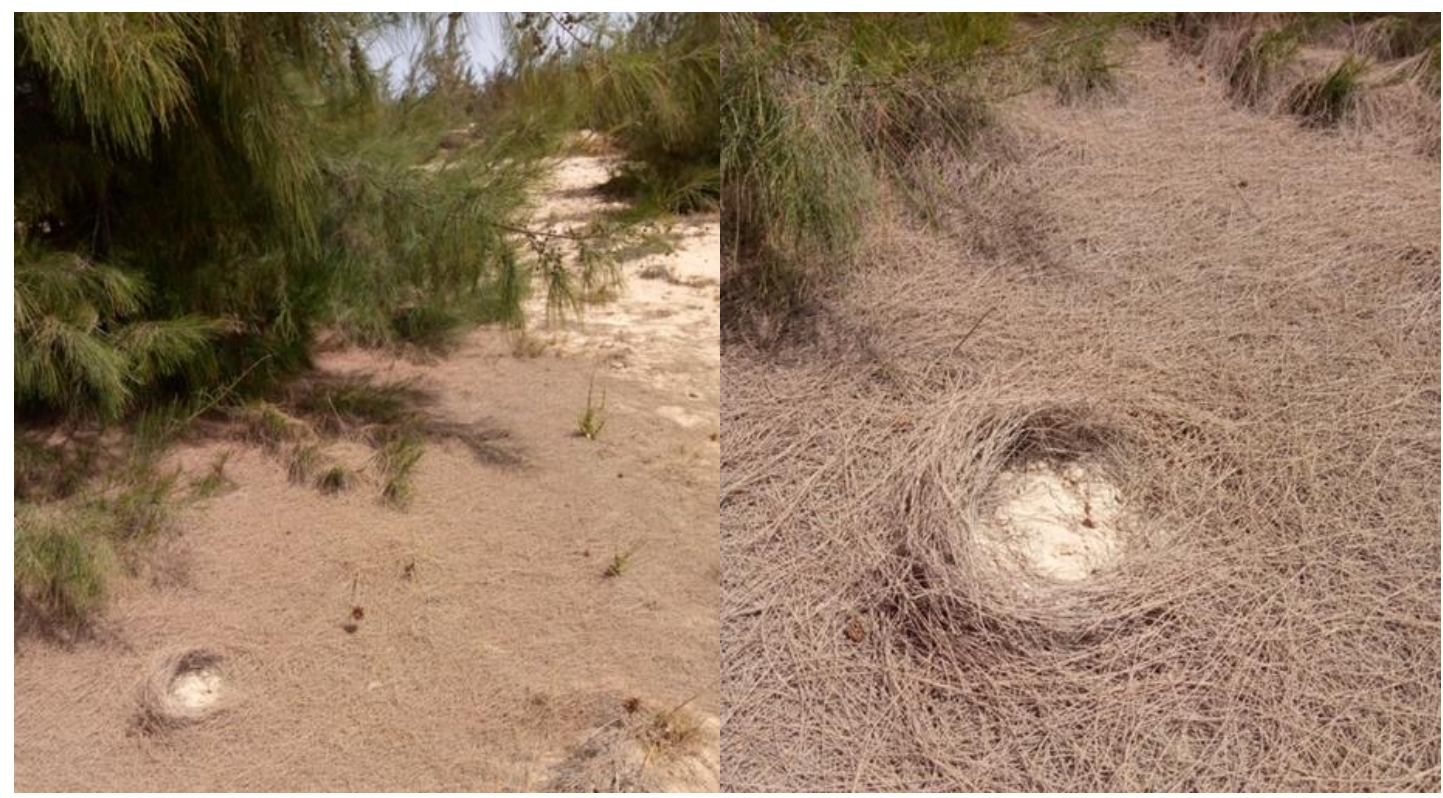

Photos 6 et 7. Tapis de litière de l'espèce Casuarina equisetifolia (Sy, 2021)

Ces réponses montrent toute l'importance de l'espèce Casuarina equisetifolia (filao) contre la dynamique d'ensablement. Elle fonctionne comme brise-vent en sus de produire un tapis de litière qui a pour effet d'inhiber la déflation, de bloquer les échanges sédimentaires entre le cordon blanc vif (qui produit des particules de quartz stériles) et les cuvettes maraichères. En conséquence, elle régénère le sol et protège les espèces cultivées contre la chaleur insupportable pour certaines cultures. Quelque 93,3 $\%$ de la population enquêtée affirment une évolution des espèces en quantité et en qualité. Pour protéger ces cultures, les populations initient des actions, des stratégies de protection des cultures. Dans ce cadre, les réponses des enquêtés en \% a donné les statistiques suivantes : néant $=53,4 \%$, Ceinture de 
l'espèce Opuntia tuna $=30 \%$, Ceinture végétale + palissade $=13,3 \%$, Entretien et Filao $=3,3 \%$.

La réponse prépondérante à $53,4 \%$ est une absence de mesures locales de protection des cultures et des habitats. Cette population se suffit de la bande de filao pour bénéficier de la protection des cultures. Les champs sont des aires organisées en plans de cultures diverses avec une faible clôture. C'est, en effet, des champs en finage qui marquent les terroirs villageois de Golgaïndé et de Bono. L'analyse du paysage agraire révèle une configuration de l'espace qui met en avant une certaine maîtrise de la menace d'ensablement sur les espaces de culture. Cette situation peut justifier l'absence de stratégies locales de protection pour plus de la moitié des personnes interrogées. Cependant, $30 \%$ de la population interrogée utilisent « une ceinture de l'espèce Opuntia tuna comme clôture des espaces de culture. C'est une mesure qui vient en appoint de la bande de filao. L'installation de cette clôture n'est pas uniquement contre l'ensablement mais elle est aussi mise en place contre les reptiles et autres animaux dangereux. Cette espèce est aussi traditionnellement associée à «une ceinture végétale et palissade » utilisées par 13,3\% des personnes interrogées. Une dernière partie de la population $(3,3 \%)$ se contente de «l'exploitation et de l'entretien de la bande de filao » qu'il réaménage et réutilise d'une manière plus adéquate à leur besoin.

Il est ressorti une meilleure lecture du degré de satisfaction des populations de Mboro concernant la protection : amélioration du rendement due à la régénération du sol, à la protection contre l'ensablement et contre la chaleur. Ce qui conduit à une production de qualité. Ces conditions agronomiques et environnementales permettent d'accroitre les revenus des maraîchers, améliorant leurs conditions de vie. Voilà tout l'enjeu de protéger ces territoires économiques (cadres de vie) exposés à la mobilité du matériau dunaire. Cette protection doit se faire avec l'appui de l'information géographique sur la dynamique des débits solides, justifiant la place de la géomorphologie littorale.

\section{Discussion}

Cette contribution vise à montrer la portée scientifique de la recherche géomorphologique en termes d'enjeux socio-économiques. Sur le risque et les enjeux des territoires, la confrontation des données avec un article sur la vulnérabilité permet la lecture de leur fiabilité. Il s'agit d'une «Étude économique et spatiale de la vulnérabilité et de l'adaptation des zones côtières aux changements climatiques» (egis international, 2013). Elle traite de la vulnérabilité côtière et procède par l'évaluation économique et spatiale des risques et des vulnérabilités pour aider à la prise de décision et à la gestion intégrée de la zone côtière. Ses conclusions sont une vulnérabilité socioéconomique établie sur le littoral sénégalais avec des projections sur plusieurs 
années (2030, 2080), la mise en place de cartes de risque avec une catégorisation en fort, moyen et faible risque sur l'espace côtier.

La dimension politique de cette étude embarque la prise de décision et la mise en place de systèmes d'alerte contre les risques. Ainsi, même si les échelles diffèrent autant que certaines approches (cartographie, vulnérabilité, projection du risque), cet article confirme la présence du risque sur le littoral Nord sénégalais à travers les échanges sédimentaires entre le cordon vif et les espaces de culture à enjeux socio-économiques multiples.

Dans ce cadre, Faye (2010) dans sa thèse de doctorat a évalué les évolutions respectives des traits de côte de plusieurs plages ouest-africaines, de la Mauritanie à la Guinée Bissau. Des mesures quantitatives sont extraites du traitement des images satellites et la photo-interprétation a permis à Faye de tirer des conclusions sur la mobilité du trait de côte ouest-africain. Les variations temporelles permettent de suivre cette évolution sur $142 \mathrm{~km}$ de plage $(7,5 \%)$ des $1892 \mathrm{~km}$ de côtes sableuses. Un pourcentage de $73,4 \%$ de cet échantillon côtier est en pro-gradation avec quelques marges d'érosion. Ces secteurs sont localisés dans les cordons littoraux, les îles sableuses estuariennes du Sénégal (Langue de Barbarie, Joal, Saloum, Casamance), de la Gambie (Toll Point, Saniang Point, Kartrung Point) et de la Guinée Bissau (Cap Roxo, Rio Cacheu, Ouest de l'île de Pexice, Nord du Rio Cacine, flèche d'Acapa, la façade orientale de l'île d'Adonga). De cette recherche il apparait que $5,5 \%$ de côtes pro-gradent, $2 \%$ de côtes s'érodant et $92,5 \%$ sont à évolutions non mesurables par les données satellites disponibles.

Ces données concordent avec l'hypothèse du milieu côtier par excellence dynamique compte tenu de la force éolienne en action, du ravivement et de la mobilité des dunes, de la sensibilité du matériau dunaire à la déflation éolienne. Dans le même sillage, Ndao (2012) a travaillé sur la gestion environnementale d'un territoire humide et dynamique qui parcourt la Grande Côte, de Saint-Louis à Dakar ; les Niayes très propices à la production horticole et particulièrement importante pour la survie des populations de Pikine à Dakar, de Mboro à Thiès et à Saint-Louis. Les résultats de cette recherche soulignent la vulnérabilité des populations par des inondations récurrentes, une occupation du sol en hausse inexorable sans mesure préventive ni de plan directeur, une précarité socio-économique de populations ancrées dans les réflexes d'aridité et de sécheresse. Il s'y ajoute une situation aléatoire du risque climatique qui fait des Niayes un écosystème à risque et exposé aux actions anthropiques, etc. Ndao a, en quintessence, évalué les aspects territoriaux des Niayes. La cartographie de l'occupation du sol de Mboro révèle le dynamisme socio-économique de ces espaces. Les résultats de cet article confirment la problématique socio-économique en cours même avec des écarts méthodologiques consistants. Les deux études ont cependant, montré les richesses agronomiques de la zone des Niayes. C'est 
d'ailleurs sous ce rapport qu'une alternative de recherche sérieuse est offerte à travers un bon nombre de travaux scientifiques sur la Grande Côte qui ont utilisé des expériences empiriques de trappes à sédiments pour quantifier ce risque morphogénique sur les Niayes : Sy (2013), Niang (2017), Thiaw (2018), Seck (2019), Seck, Sy et Sy (2020). Ces travaux ont produit des statistiques sur les quantités de débits massiques éoliens mobilisés par la déflation éolienne et permettent de témoigner de ce risque d'ensablement présent dans la Grande Côte du Sénégal.

En définitive, les données présentées dans cette contribution trouvent une validité probante au regard de nombreux résultats issus de travaux scientifiques avérés. Cependant, l'analyse de la dynamique de l'ensablement, au-delà de la recherche de la sensibilité granulométrique des sédiments, peut être quantifiée par des méthodes de terrain spécifiques à la géomorphologie littorale.

\section{Conclusion}

Un seuil critique des vents compétents combiné à une granulométrie moyenne à $80 \%$, fine et très fine (autour de $12 \%$ ), un déficit pluviométrique avéré qui assèche les sédiments dépourvus de liants, un système dunaire très mobile, sont autant de facteurs qui favorisent la mise en place d'un système morphogénique à dominante mécanique : la morphodynamique éolienne. Ces éléments expliquent la dynamique érosive (Charles, 2010), véritable menace dans le secteur côtier de Mboro. Ces menaces se mutant en risque réel en présence de facteurs de production socio-économiques, les enjeux autour des territoires de Mboro interpellent la recherche actuelle. Le littoral est « un espace de vie »(Miossec, 1998) plutôt attrayant pour les populations sénégalaises dont plus de la moitié vit à moins de $60 \mathrm{~km}$ des côtes. C'est une mosaïque de territoires qui se superposent et des mutations spatiales caractérisent ces écosystèmes littoraux. La mise en place de données exhaustives sur la dynamique de l'ensablement offrent un support important pour l'aménagement du territoire en dehors de la recherche purement scientifique. C'est en d'autres termes, une dynamique de gestion territoriale d'un milieu aménagé d'une bande de filao pour la lutte contre l'ensablement des espaces maraîchers.

\section{References:}

1. Charles Jacques Berthe, Moustapha Ould Mohamed \& Meimine Ould Salek (2010). Lutte contre l'ensablement, l'exemple de la Mauritanie, FAO, 89 pages.

2. Fall Mamadou (1986). Environnements sédimentaires quaternaires et actuels des tourbières des Niayes de la Grande Côte du Sénégal. Thèse 
de doctorat de $3^{\text {ème }}$ cycle, Université Cheikh Anta Diop de Dakar, Géologie, 153 pages.

3. Faye Ibrahima (2010). Dynamique du trait de côte sur les littoraux sableux de la Mauritanie à la Guinée-Bissau (Afrique de l'Ouest) : Approche régionale et locale par photo-interprétation, traitement d'images et analyse de cartes anciennes. Thèse de doctorat, Université de Bretagne Occidentale, Volume 1, 321 pages.

4. Fournier Jérôme, Chantal Bonnot-Courtois, Raphaël Paris \& Monique Le Vot (2012). Analyse granulométrique, principes et méthodes. CNRS, Dinard, 99 pages.

5. Miossec Alain (1998). «Les littoraux espaces de vie » in Dossiers des images économiques du monde, sous la dir. de Gamblin, A., édition SEDES, pp 41-59.

6. Myriam Olivier \& Ali Mesbah (2016). «Construction en terre», Essais d'identification des sols. Cayenne, Guyane, 45 pages.

7. Ndao Mariétou (2012). Dynamiques et gestions environnementales de 1970 à 2010 des zones humides au Sénégal : étude de l'occupation du sol par télédétection des Niayes avec Djiddah Thiaroye Kao (à Dakar), Mboro (à Thiès) et Saint-Louis. Thèse de doctorat, Université de Toulouse 2 Le Mirail, cotutelle internationale Université Gaston Berger de Saint-Louis, 371 pages.

8. Niang Souleymane (2017). Dégradation chimique et mécanique des terres agricoles du Gandiolais (littoral nord du Sénégal) et analyse des dynamiques actuelles d'adaptation. Thèse de doctorat de géographie, Université Gaston Berger de Saint-Louis, 357 pages.

9. Seck Mouhamadou Bassirou (2019). Dynamique de l'ensablement et protection des Niayes de Mboro. Quantification des débits solides éoliens et cartographie des aménagements de protection. Thèse de doctorat de géographie, Université Gaston Berger de St-Louis, 238 pages.

10. Sy Amadou Abou (2009). Les dunes littorales sénégalaises. Dynamique actuelle et ses conséquences sur les espaces maraîchers. Mémoire de master 2, UFR LSH, UGB, 193 pages.

11. Sy Amadou Abou (2013). Dynamique sédimentaire et risques actuels dans l'axe Saint-Louis-Gandiol, littoral Nord du Sénégal. Thèse de doctorat de géographie, Université Gaston Berger de St-Louis, 333 pages.

12. Tangara Abdourahmane (2010). «Données anémométriques et charriage de sable sur la côte nord du Sénégal, cas des secteurs de Kayar et de Mboro », in Revue de Géographie du Laboratoire Leïdi, $\mathrm{n}^{\circ} 08$, p. 145-153. 
13. Thiaw Pape (2018). Dynamique de l'ensablement des Niayes et ses conséquences sur le maraichage dans le secteur de Potou: stratégies de lutte. Mémoire de Master 2, Université Cheikh Anta Diop de Dakar, Sénégal, 178 pages. 